\title{
Egressos de enfermagem e suas concepções sobre o mundo do trabalho
}

\author{
Nursing graduates and theirs notions of the world of work \\ Egresados de enfermería y sus concepciones sobre el mundo del trabajo
}

\author{
Carolina Viegas de Mello' ${ }^{\prime}$; Shino Shojil ${ }^{\circ}$; \\ Norma Valéria Dantas de Oliveira Souzal ${ }^{\circ}$; Célia Regina da Silva Medeiros ${ }^{\prime \prime}$ | \\ 'Universidade do Estado do Rio de Janeiro, Rio de Janeiro, RJ, Brasil; \\ "Universidade Federal do Rio de Janeiro, Rio de Janeiro, RJ, Brasil
}

\begin{abstract}
RESUMO
Objetivo: identificar e analisar os aspectos facilitadores e limitadores dos graduandos de enfermagem em sua inserção profissional. Método: estudo qualitativo e descritivo, desenvolvido em duas universidades federais do Rio de Janeiro. Os participantes foram 43 egressos dessas universidades, no qual foi aplicado um roteiro de entrevista semiestruturada. 0 tratamento dos dados ocorreu pela análise lexical com auxílio do software Iramuteq. Resultados: constatou-se que, na Universidade A, os fatores facilitadores para a execução do trabalho foram autonomia, relacionamento interpessoal e equipe de trabalho. Os limitadores caracterizam-se como sobrecarga de trabalho, escassez de recursos humanos e de materiais, qualificação profissional e baixo reconhecimento. Na Universidade B, os fatores facilitadores foram condições laborais, trabalho em equipe e autonomia. Já os limitadores foram sobrecarga de trabalho, déficit de recursos humanos e de materiais e relacionamento interpessoal. Conclusão: ressalta-se que os fatores dificultadores suplantaram os facilitadores e são necessárias intervenções e reformulações não apenas no ambiente de trabalho, mas também no campo da formação.
\end{abstract}

Descritores: Trabalho; Ambiente de Trabalho; Educação em Enfermagem.

\section{ABSTRACT}

Objective: to identify and examine the conditions facilitating and limiting nursing students' integration into work. Method: in this qualitative and descriptive study conducted at two federal universities in Rio de Janeiro, forty-three graduates from these institutions were our focal group, to whom a semi-structured interview script was applied. The data collected were subjected to lexical analysis, assisted by Iramuteq software. Results: at University A, the factors facilitating nursing work were: autonomy, interpersonal relations and the nursing team. Limiting aspects were: overwork, shortage of human and material resources, professional qualification and lack of recognition. At University B, the facilitating factors were: working conditions, teamwork and autonomy. Limiting conditions were: shortage of human and material resources and interpersonal relations. Conclusion: limiting aspects far outweighed those facilitating nursing work, and interventions and reformulations are necessary not only in the workplace, but also in the field of training.

Descriptors: Work; Working Environment; Education Nursing.

\section{RESUMEN}

Objetivo: identificar y analizar los aspectos facilitadores y limitantes de los estudiantes de enfermería en lo relativo a su inserción profesional. Método: estudio cualitativo y descriptivo, desarrollado en dos universidades federales de Río de Janeiro. Los participantes fueron 43 egresados de estas universidades, en los que se aplicó un guion de entrevista semiestructurada. El tratamiento de datos se realizó mediante análisis léxico con la ayuda del software Iramuteq. Resultados: se verificó que, en la Universidad A, los factores facilitadores para la realización del trabajo fueron: autonomía, relaciones interpersonales y equipo de trabajo. Los limitadores se caracterizan por: sobrecarga de trabajo, escasez de recursos humanos y de materiales, calificación profesional y poco reconocimiento. En la Universidad B, los factores facilitadores fueron: condiciones de trabajo, trabajo en equipo y autonomía. Los limitadores fueron: sobrecarga de trabajo, escasez de recursos humanos y de materiales, y relaciones interpersonales. Conclusión: cabe señalar que los factores dificultadores sobrepasaron los facilitadores e intervenciones y reformulaciones son necesarias, no solo en el ámbito laboral, sino también en el campo de la formación.

Descriptores: Trabajo; Ambiente de Trabajo; Educación en Enfermería.

\section{INTRODUÇÃO}

O objeto deste estudo é a percepção de egressos da graduação em enfermagem sobre o mundo do trabalho e o seu ingresso no ambiente laboral, sendo elencado como objetivo identificar e analisar os aspectos facilitadores e limitadores constatados pelos graduandos de enfermagem em sua inserção profissional.

O trabalho de enfermagem vem sofrendo várias repercussões com a globalização e com a implantação da política neoliberal no contexto nacional. Tais fenômenos tem provocado a precarização laboral, com a crescente terceirização e retração do quantitativo de trabalhadores atuantes em regime formal; prejudicando a garantia dos direitos sociais, aumentando o desemprego e o subemprego, como também elevando a rotatividade da força de trabalho. Esta situação 
resulta em prejuízos para a qualidade do cuidado de enfermagem e para a saúde dos trabalhadores, bem como para o processo de inserção profissional dos egressos de enfermagem ${ }^{1}$.

Entretanto, é necessário inferir mudanças positivas, como no campo científico, ocorridas nas últimas três décadas, responsáveis pelo impacto no desenvolvimento do conhecimento na área de Enfermagem. As mesmas acompanharam as tendências mundiais, refletindo os avanços necessários relacionados às ações de cuidado, de ensino e às demandas de pesquisa na área de Enfermagem ${ }^{2}$. Corrobora-se, também, os comprometimentos políticos com debates junto às instâncias competentes, frente à conquista de um piso salarial para a profissão e de uma jornada de trabalho semanal de 30 horas $^{3}$ (Projeto de Lei 2295/00), ressaltando o processo de fortalecimento da Enfermagem quanto às condições laborais dignas e inovadoras.

Frente a esse cenário, considera-se a problemática vinculada ao mundo do trabalho multifacetada e complexa, e certamente, uma das recomendações para transformá-la dá-se por meio da formação. Este estudo respalda-se no princípio de que é pela formação profissional que se pode transformar a realidade do trabalho em saúde, sob uma perspectiva freireana, uma vez que "educação não transforma o mundo. Educação muda pessoas. Pessoas transformam o mundo" ${ }^{\prime \prime}$, corroborando que a educação pode ser considerada como importante ferramenta de mudança e transformação social do homem.

Neste sentido, faz-se relevante que os currículos institucionais sejam sistematicamente avaliados, num processo dinâmico de construção e (re)construção, com o objetivo de preparar os futuros enfermeiros, tornando-os em agentes transformadores, por meio de um processo ensino-aprendizagem reflexivo, crítico e problematizador, aproximando os estudantes sistematicamente dos campos de atuação da profissão ${ }^{5-7}$.

Por outro lado, estudos ${ }^{4}$ inferem que os egressos de enfermagem se deparam com profundo estranhamento quanto à configuração do mundo do trabalho. Tal estranhamento provém à conta da dinamicidade e complexidade que se empreendeu nos espaços laborais a partir da globalização e do modelo neoliberal. $E$, frente essa realidade, indo de encontro ao que lhes foi ensinado na academia, é comum que esses profissionais entrem em conflito e apresentem sofrimento psíquico ${ }^{8,9}$. Considera-se ${ }^{10,11}$, portanto, fundamental a proposta curricular que trabalhe a inserção precoce desses estudantes nos cenários de práticas, oportunizando o contato com os desafios singulares da profissão.

Sendo assim, frente a esses fatores, levantaram-se os seguintes questionamentos: Como se apresenta o mercado de trabalho para o enfermeiro recém-formado? Quais são as facilidades e os desafios que o egresso de enfermagem enfrenta após sua inserção profissional?

\section{REFERENCIAL TEÓRICO}

A abertura indiscriminada de cursos de graduação, assim como a alocação desequilibrada de enfermeiros no território nacional, gerou desdobramentos negativos para a profissão e para os trabalhadores, verificando-se elevado subemprego; trabalhadores cooperativados e/ou terceirizados, direitos trabalhistas comprometidos; péssimas condições de trabalho; elevada carga horária laboral e severa e excludente competitividade profissional ${ }^{12}$.

O ensino de Enfermagem no Brasil também sofreu reflexos dos momentos sócio-históricos, evidenciando a relevância de superar a desvinculação que se estabeleceu entre o processo de formação e a realidade praticada, propondo-se então formar enfermeiros capazes de atender as demandas de saúde da população, atuando de forma dinâmica e em consonância com os princípios da universalidade, integralidade e equidade ${ }^{13}$. A proposta curricular oficializada pela Portaria $n^{\circ} 1.721 / 1994^{14}$ pauta-se na educação como possibilidade de transformação, fundamentandose na construção de um potencial crítico-reflexivo, com a sociedade, buscando qualidade de assistência prestada e crescimento da profissão.

Pesquisas revelam a preocupação quanto às relações entre a prática educativa das instituições formadoras e a realidade do profissional enfermeiro para desempenhar o seu papel nos serviços de saúde ${ }^{15,16}$.

Faz-se necessário induzir mudanças na formação desses profissionais, por meio da construção de novos projetos político-pedagógicos que os subsidiem a lidarem com os desafios do exercício profissional em um contexto laboral de constantes mudanças ${ }^{14}$; e viabilizem também um ensino vinculado ao mundo do trabalho e à realidade cotidiana das práticas sociais, propiciando o desenvolvimento de habilidades e competências de um modo multidisciplinar, cultural, científico e técnico ${ }^{17}$.

\section{MÉTOdo}

Adotou-se uma abordagem qualitativa e descritiva para o desenvolvimento do estudo. Os campos de pesquisa foram duas faculdades de enfermagem de caráter federal, ambas localizadas no Rio de Janeiro, classificadas como Universidade A e Universidade B. 
Os critérios de inclusão de participantes no estudo foram: a) que trabalhassem na profissão há pelo menos um ano, e b) que tenham se graduado durante entre 2000 e 2013, com objetivo de captar o cenário dos egressos na perspectiva desse último milênio.

Sobre o quantitativo de participantes, planejou-se coletar dados com um graduado de cada turma, perfazendo um total de 56 participantes, ou seja, quatro egressos por ano, haja vista que cada instituição ofereceu dois ingressos de estudantes por ano letivo. A escolha desses egressos foi aleatória, feita por meio de sorteio. Ao final do processo de coleta, obteve-se um total de 43 participantes. Esse quantitativo reduzido em relação ao planejado inicialmente deu-se por falta de dados cadastrais dos egressos, recusas de participação e falta de feedbacks aos convites contribuírem na coleta de dados. Salienta-se que a escolha dos participantes do estudo não se prendeu ao quantitativo dos mesmos e sim, ao conhecimento e/ou vivência destes trabalhadores acerca do objeto de estudo. Nesta perspectiva, defende-se que o número de participantes na pesquisa qualitativa é um parâmetro pouco valorizado, uma vez que o que o objetivo é o conteúdo levantado para apreensão do objeto da investigação ${ }^{18}$.

Corroborando, em uma metodologia de base qualitativa, o número de participantes que compõe o quadro de entrevistas dificilmente pode ser anteriormente determinado, dependendo assim, da qualidade das informações obtidas em cada depoimento, assim como do grau de recorrência e divergência destas informações. Enquanto estiverem aparecendo "informações" originais ou pistas que possam indicar novas perspectivas à investigação em curso, as entrevistas devem continuar sendo realizadas ${ }^{19}$.

O instrumento de coleta utilizado foi um roteiro de entrevista semiestruturada, aplicada de junho a setembro de 2016. E para tratamento dos dados, optou-se pela análise lexical ou textual. Foram aplicados métodos estatísticos aos materiais verbais transcritos, permitindo explorações minuciosas e viabilizando a construção de categorias naturais a partir de algumas técnicas estatísticas no campo de dados qualitativos ${ }^{20}$. Esse tipo de análise tem como unidade o vocabulário: as palavras são identificadas e quantificadas em termos de frequência, priorizando assim a sistematização dos dados textuais para somente depois interpretá-los ${ }^{21}$.

Fundamentando-se nessa perspectiva, após a transcrição individual do conteúdo de cada entrevista , realizou-se a análise textual com auxílio do software Iramuteq (Interface de R pour les Analyses Multidimensionsionnelles de Textes et de Questionnaires), um programa livre ancorado no software R (pacote gratuito para realizar análises estatísticas) e que possibilita diferentes processamentos e análises estatísticas dos textos inseridos ${ }^{22}$. 0 software permitiu dois tipos de interpretação de resultados para esta pesquisa: a de frequência e a de rede de palavras.

A frequência de palavras, ou lexicografia básica, é um indicador que apresenta a contagem das palavras presentes no texto analisado. Nesta etapa, são observadas as palavras mais frequentes do texto, deduzindo que determinado vocábulo frequentemente repetido seja pertinente no contexto investigado ${ }^{23}$; assim, é possível encontrar itens passíveis de categorização. Os dados foram então submetidos ao software Iramuteq e tratados à luz da análise temática de conteúdo. Emergiu daí a seguinte categoria: Contexto do trabalho em saúde: aspectos facilitadores e limitadores da atuação dos egressos.

Atendendo aos preceitos éticos, esta pesquisa foi cadastrada na Plataforma Brasil, conforme preconizado pela Resolução 466/2012, do Conselho Nacional de Saúde (CNS/MS), obtendo aprovação do Comitê de Ética sob protocolo no 1.293.828, e Certificado de Apresentação para Apreciação Ética em Pesquisa (CAAE) sob o número 49971815.5.0000.5282.

Para manter o anonimato e preservar a privacidade dos egressos, foi utilizada uma codificação para cada entrevista. Assim, foram atribuídos os códigos $A 1, A 2, A 3, B 1, B 2$ e B3, e assim sucessiva e respectivamente, de acordo com a universidade de origem do entrevistado e a ordem cronológica do preenchimento das entrevistas.

\section{RESULTADOS E DISCUSSÃo}

Em relação às 21 entrevistas analisadas na Universidade A, obtiveram-se três importantes temáticas: autonomia profissional, bom relacionamento profissional e equipe de trabalho.

Em relação à autonomia profissional (seis ocorrências, $29 \%$ do total), aspecto apontado por egressos de ambas as universidades como facilitador do processo laboral, ressaltou-se a importância de um posicionamento respeitoso e de valorização dos egressos por parte da equipe de enfermagem e dos demais profissionais da área. Um ambiente com essa configuração favorece que o enfermeiro tenha prazer e se sinta apoiado para desempenhar suas atribuições, fortalecendo a autonomia.

Outro aspecto facilitador enfatizado pelos participantes da Universidade A foi o bom relacionamento interpessoal com os demais membros da equipe de saúde (sete ocorrências, 33\%), bem como a presença de uma comunicação eficiente. Esses dados enfatizaram a importância do coletivo para a satisfação do egresso. 
Mas as facilidades é que a gente tem um bom relacionamento, todas as equipes se dão muito bem. Todos conseguem dialogar cordialmente, e por conta desse diálogo se consegue facilitar a atuação profissional (A2).

Do mesmo modo, a quantidade e a qualificação satisfatórias dos profissionais, para a execução da assistência, constituíram um grande facilitador para a execução das atividades (uma ocorrência, 5\%):

Tem sempre dois enfermeiros e cinco técnicos que estão de plantão. Então você trabalhar com outro enfermeiro, ajuda se você está com uma limitação ou uma dificuldade, você tem uma dupla para trabalhar. São profissionais mais qualificados para estarem exercendo sua função (A17).

O dimensionamento de pessoal é um fator de suma importância para execução das atividades, uma vez que evita a sobrecarga de trabalho e, consequentemente, o desgaste da equipe de enfermagem, garantindo, portanto, qualidade na prestação de assistência ${ }^{24}$.

Já em relação à Universidade B, 22 egressos participaram da entrevista; após análise com auxílio do software Iramuteq, elencam-se três importantes temáticas: condição laboral favorável; trabalho em equipe e autonomia laboral.

Quanto ao local de trabalho, três egressos ( $14 \%$ do total) correlacionaram a disponibilidade de materiais com as facilidades da estrutura física e dos recursos humanos, alegando que um trabalho eficaz depende de uma boa estrutura e de profissionais bem qualificados.

Para quem gosta e atua na assistência, é ótimo trabalhar tendo todo equipamento, monitor completo, eletrodo, saturímetro, bomba de seringa, que em pediatria é muito usado, bomba de roldanas sempre com atualização (B4).

Paralelamente, o trabalho em equipe foi o facilitador mais mencionado em ambos os grupos de egressos, citado por metade de cada um deles: 11 da Universidade A (50\%) e 11 da Universidade B (50\%). Todos os egressos citaram um bom relacionamento com a equipe multidisciplinar, com a equipe de enfermagem e com a própria categoria profissional como um todo.

Como é uma equipe multidisciplinar, isso é uma facilidade para gente, porque é uma equipe voltada ao atendimento para o paciente de forma integral. Temos contato muito próximo com outros profissionais que complementam nosso atendimento, e isso é bom para a enfermagem, pois o paciente melhora, e nós crescemos como profissionais (B15).

O trabalho em equipe, sendo um elemento fundamental nas instituições de saúde, é o meio mais adequado para se obter melhores resultados; os objetivos congregam-se para alcançar a excelência na qualidade do trabalho desempenhado, gerando melhores soluções de problemas, além de desenvolver e executar ações impossíveis de serem realizadas sozinhas ${ }^{25}$.

Quando se fala em trabalho em equipe, é relevante mencionar o bom relacionamento profissional, aspecto aludido por egressos da Universidade A. Uma dinâmica cooperativa e generosa entre os membros da equipe é um poderoso instrumento a ser usado em prol do bom desempenho profissional e da prestação adequada de assistência ao paciente. Gera-se, assim, prazer na execução do trabalho, protegendo e assegurando a subjetividade do trabalhador ${ }^{26}$.

Cinco egressos da Universidade B (23\%) estão satisfeitos com a autonomia conquistada através do apoio na tomada de decisões e da liberdade promovida para suas respectivas atuações, salientando que o coletivo profissional Ihes dá confiança em relação às condutas prescritas.

Eu tenho muita facilidade em exercer o meu papel pois é uma equipe que me apoia e acredita em meu trabalho. A equipe de enfermagem me entende, a chefia me dá autonomia para muitas coisas dentro da minha unidade (B21).

Sabe-se que a autonomia conferida ao indivíduo em suas funções oferece confiança para a prestação do serviço, além de se tornar uma boa ferramenta para produtividade, pois o profissional trabalha mais e melhor, o que diretamente implica em qualidade dos cuidados prestados. Também confere proteção a subjetividade do trabalhador, pois reverte-se em satisfação no desempenho das tarefas ${ }^{27}$.

Em relação aos aspectos limitadores, as correlações que emergiram da análise das falas dos 21 egressos da Universidade A, se deram entre a sobrecarga de trabalho, a escassez de recursos humanos e de materiais, a desorganização do processo laboral, a má qualificação profissional da equipe de enfermagem e o pouco reconhecimento profissional, objetos que entraram em contradição com as facilidades mencionadas anteriormente.

A sobrecarga de trabalho foi apontada por cinco egressos ( $24 \%$ do total), sendo principalmente relacionada à falta de funcionários, ao grande quantitativo de pacientes e à elevada carga de trabalho.

Dificuldades é com relação aos pacientes, pois são 26 pacientes para só um enfermeiro plantonista. Também com relação à gravidade destes pacientes, acho que é pouco enfermeiro para desempenhar as funções devidas. Em relação à equipe de técnicos, também são poucos. Hoje eu tenho vinte e seis pacientes e só quatro técnicos. Eles, ficando sobrecarregados, eu também fico. Toda a equipe fica sobrecarregada (A10). 
A sobrecarga de trabalho foi um tópico levantado por egressos de ambas as Universidades. Resultado das novas formas de gestão relacionadas ao ideário neoliberal, a sobrecarga acarreta mais precariedade ao ambiente laboral por meio da polivalência e da multifuncionalidade do trabalhador, que, por sua vez, se sente pressionado a atingir as metas impostas pela organização do trabalho e, sobretudo, para executar o trabalho com qualidade na intenção de oferecer o melhor ao paciente ${ }^{28}$

A falta de recursos humanos também foi citada por sete participantes (33,33\% do total) como limitador para execução das atividades laborais, com consequente sobrecarga de trabalho dos profissionais. Assim, essa falta é um elemento comprometedor da qualidade da assistência prestada.

Além da insuficiência de recursos humanos, sete egressos (33\% do total) relataram a escassez de recursos materiais, como exemplificado no discurso destacado a seguir:

A falta de material é complicada, dificulta a gente de trabalhar. Então, assim, falta material, e você fica como? Eu preciso de material para trabalhar, eu sozinha com a minha equipe não faço nada (A21).

$\mathrm{O}$ déficit de recursos materiais e humanos é outro fator que aumenta a demanda laboral e o ritmo de trabalho. A inadequação de recursos e de condições de trabalho, também relacionada ao contexto neoliberal, além de causar sofrimento psíquico, faz com que os profissionais modifiquem seu modus operandi, o que altera a dinâmica laboral por meio da incorporação de adaptações e improvisações cujo objetivo é, em última instância, suprir a carência de materiais para prestar o cuidado ${ }^{29}$. Trata-se, portanto, de um processo vicioso, no qual as maiores consequências acabam por desencadear, com frequência, a baixa qualidade de assistência de saúde prestada ao cliente.

Destacou-se também a inadequação do processo de trabalho, aludida por cinco egressos ( $24 \%$ do total):

Falta de organização de alguns processos que dificultam o trabalho. Não há planejamento, tudo é improvisado (A6).

Outro fator dificultador levantado por cinco egressos (24\%) foi a qualificação profissional inadequada da equipe de enfermagem, aspecto mais mencionado por egressos que atuam em instituições hospitalares públicas, nas quais o regime empregatício é estatutário e garante, portanto, a empregabilidade do profissional mesmo quando este já está desatualizado técnica e cientificamente.

Existe uma carência muito grande de conhecimento técnico de muitos profissionais, tanto de técnicos quanto de enfermeiros. Então, eu entendo que, talvez até pelo fato de não terem um domínio grande em relação a determinados procedimentos, implica em um déficit no próprio cuidado de enfermagem aos pacientes (A8).

Corroborando, os egressos relataram que seus pares, de um modo geral, não buscam aprimoramento e capacitação. Essa defasagem gera um decréscimo na qualidade da assistência de enfermagem, bem como uma mentalidade mais resistente a mudanças positivas no processo laboral por parte desses profissionais. Essa condição agrava, cada vez mais, o quadro da desvalorização da enfermagem e o da falta de reconhecimento profissional e social ${ }^{30}$.

Outro importante dado constatado por dois entrevistados (9\%) foi a ausência de reconhecimento dos egressos no local de trabalho.

Ao conversar com colegas que trabalham na ponta, independente da formação profissional, percebo que o modelo de gestão atuante no nosso município, que prioriza a valorização do profissional de acordo com o alcance de metas, não percebe o trabalhador enquanto um ser que apresenta outras necessidades em seu serviço, e que a discussão sobre desenvolver o trabalho está renegada a último plano (A28).

É fundamental que os gestores de recursos humanos identifiquem e valorizem o potencial individual de cada colaborador, já que cada indivíduo possui idéias e visões diferentes, para, assim, somar com as potencialidades de outros colaboradores da equipe de trabalho, resultando em melhor qualidade do produto ou serviço que se está ofertando à sociedade ${ }^{25}$. Ao mesmo tempo, valorizar tais aspectos individuais dentro de uma equipe promove e protege a saúde do trabalhador, valorizando-o dentro da dinâmica laboral ${ }^{31,32}$.

Já quanto à Universidade B, a partir da análise das entrevistas dos 22 egressos, apresentaram-se aspectos limitadores semelhantes às da Universidade A, correlacionados às seguintes temáticas: sobrecarga de trabalho; falta de condições de trabalho (recursos humanos e materiais) e mau relacionamento entre os funcionários.

No contexto do local de trabalho, metade dos egressos $(11 ; 50 \%)$ relatou sobrecarga de trabalho, destacando que degaste físico e emocional, superlotação e pacientes de alta complexidade são alguns dos gatilhos.

E no contexto de sobrecarga de trabalho e desgaste, faltam infraestrutura física e recursos materiais (mencionada por seis egressos; $27 \%$ ), além de recursos humanos ( $7 ; 32 \%)$, constatada pela fala a seguir:

Hoje é a falta de funcionários, excesso de trabalho e multitarefas, mas a falta de funcionário é o que mais impacta. Muitas vezes deixamos de fazer o papel de enfermeira para fazer o papel de técnico, devido à falta de técnico (B13). 
Nesse ínterim, constata-se que para a Universidade B, a estruturação das condições para o trabalho é relevante para o bom desempenho laboral. O local de atuação deve estar satisfatoriamente projetado (condições de higiene, segurança e equipamentos) a fim de garantir conforto a toda a equipe, proporcionando saúde e segurança para a execução de suas atividades. Embora ainda muito banalizada por empregadores e administradores, essa medida é essencial para diminuir a incidência de morbidades ocupacionais e para melhorar a qualidade da assistência prestada ${ }^{33,34}$.

Dentre os problemas relacionados aos recursos humanos, três egressos (14\%) manifestaram insatisfação com o relacionamento interpessoal, conforme exemplificado a seguir:

O relacionamento interpessoal entre os membros da equipe é muito ruim, péssimo. [...] Não, não é unido. Pelo contrário, é arma apontada um para o outro o tempo todo. É uma cultura que tem aqui (B1)

Tal situação pode ser consequência de uma estrutura hierárquica institucional, da maneira como os profissionais enfrentam suas adversidades e da resolutividade de suas dificuldades. Diferenças e divergências pessoais criam um ambiente desarmônico e geram relações profissionais deficientes, desde os líderes até os liderados, refletindo no grupo como um todo. Para contornar o problema, deve-se buscar o aprimoramento das relações profissionais, pautando-as no princípio da boa comunicação, sem deixar, porém, de considerar as peculiaridades humanas ${ }^{31}$.

\section{CONCLUSÃO}

Por meio da análise das entrevistas foi possível identificar que, na visão dos egressos, existem mais fatores limitadores do que facilitadores no local de atuação profissional, e que tais dificuldades emergem não pelo objeto de trabalho da enfermagem, mas pela configuração da organização laboral, fundamentada, no momento atual, em preceitos neoliberais.

A estrutura oferecida para o desenvolvimento das atividades laborais causa estranhamento nos profissionais. São necessárias, portanto, intervenções e reformulações não apenas no ambiente de trabalho, mas também no campo da formação, para que egressos dos cursos de graduação auxiliem na transformação deste contexto insólito, uma vez que é pela formação profissional que se pode transformar a realidade do trabalho em saúde. As instituições de ensino devem desenvolver um processo ensino-aprendizagem que fomente a reflexão, a crítica, a militância política, entre outros aspectos técnico-científicos, a fim de transformar as incongruências que vêm assolando os serviços de saúde.

\section{REFERÊNCIAS}

1. Souza NVDO, Gonçalves FGA, Pires AS, David HMSL. Neoliberalist influences on nursing hospital work process and organization. Rev. bras. enferm. [Internet]. 2017 [cited 2019 May 20]; 70(5):912-9. DOI: https://doi.org/10.1590/0034-7167-2016-0092.

2. Barros ALBL, Nóbrega MML, Santos RS, Cézar-Vaz MR, Pagliuca LMF. Research in nursing and modification of the knowledge tree in CNPq: contribution to science. Rev. Bras. Enferm. [Internet]. 2020 [cited 2020 Nov 05]; 73(1):20170911. DOI: http://dx.doi.org/10.1590/0034-7167-2017-0911.

3. Silva JL, Machado DM. Brazilian nursing in 90 years of associative history: contributions of the Brazilian Nursing Association. Hist. enferm. Rev. eletrônica. [Internet]. 2018 [cited 2020 Nov 05]; 9(2):131-40. Available from: http://here.abennacional.org.br/here/v9/n2/a4.pdf.

4. Freire, P. Pedagogia do Oprimido. Rio de Janeiro: Paz e Terra; 1987.

5. Bortoletti APG, Duarte ERM, Ferla AA, Ferreira GE, Dias MTG. Monitors' perception on education program for interprofessional healthcare work - PET-health. Esc Anna Nery. [Internet]. 2016 [cited 2020 Nov 10]; 20(2):254-60. Available from: http://www.scielo.br/scielo.php?script=sci_arttext\&pid=S1414-81452016000200254.

6. Carbogim FC, Oliveira LB, Mendonça ET, Marques DA, Friedrich DBC, Püschel VAA. Teaching critical thinking skills through problem based learning. Texto Contexto - Enferm. [Internet]. 2017 [cited 2020 Nov 10]; 26(4):e1180017. DOI: http://dx.doi.org/10.1590/0104-07072017001180017.

7. Vila V, Zhuang J, Tan E, Thorne S. Reflections on Nursing Educational Advancement Within Diverse and Evolving National Cultural Contexts. Int. J. Nurs. Educ. Schol. [Internet]. 2018 [cited 2020 Nov 10]; 15(1):e20180017. DOI: https://doi.org/10.1515/ijnes-2018-0017.

8. Püschel VAA, Costa D, Reis PP, Oliveira LB, Carbogim FC. Nurses in the labor market: professional insertion, competencies and skills. Rev. bras. enferm. [Internet]. 2017 [cited 2019 Aug 23]; 70(6):1220-6. DOI: https://doi.org/10.1590/0034-7167-20160061.

9. Silva LM, Santana TCP, Silva LRFG, Rocha LM, Canhoto CTS, Dantas KL, et al. Supervised curricular stage: difficulties and perspectives enhanced by academics of nursing. Revista Eletrônica Acervo Saúde. [Internet]. 2019 [citado em 03 Set 2019]; 18:e662. DOI: https://doi.org/10.25248/reas.e662.2019.

10. Powers S, Claus N, Jones AR, Lovelace MR, Weaver K, Watts P. Improving Transition to Practice: Integration of Advanced Cardiac Life Support Into a Baccalaureate Nursing Program. J. Nurs. Educ. [Internet]. 2019 [cited 2019 Sep 03]; 58(3):182-4. DOI: https://doi.org/10.3928/01484834-20190221-11. 
11. Browne CA, Fetherston CM. How do we facilitate international clinical placements for nursing students: A cross-sectional exploration of the structure, aims and objectives of placements. Nurse Educ Today[Internet]. 2018 [cited 2019 Sep 03]; 66:1-7. DOI: 10.1016/j.nedt.2018.03.023

12. Soares LS, Silva GRF. Health, work and neoliberalism: understanding the illness of the nursing staff. Rev. Enferm. UFPI. [Internet]. 2014 [cited 2019 Sep 03]; 3(1):141-4. Available from: http://www.repositorio.ufc.br/bitstream/riufc/10907/1/2014_art_grfsilva.pdf

13. Corbellini VL, Santos BRL, Ojeda BS, Gerhart LM, Eidt OR, Stein SC, et al. Linkages and challenges in the training of professional nurses. Rev. bras. enferm. [Internet]. 2010 [cited 2019 Sep 17]; 63(4):555-60. DOI: https://doi.org/10.1590/S003471672010000400009.

14. Presidência da República (Brasil). Portaria no 1721, de 15 de dezembro de 1994. Fixa os mínimos de conteúdo e duração do curso de graduação em enfermagem. [Internet]. Diário Oficial da União. 15 de dezembro de 1994. [cited 2019 Nov 20]. Available from: http://www.lex.com.br/doc_11078549_portaria_n_1721_de_15_de_dezembro_de_19

15. Chrizostimo MM, Brandão AAP. The training professional of nurses: 'state of the art'. Enferm Global. [Internet]. 2015 [cited 2020 Nov 08]; 40:430-45. Available from: http://revistas.um.es/eglobal/article/viewFile/208841/181721

16. Carbogim FC, Friedrich DBC, Püschel VAA, Oliveira LB, Nascimento HR. Paradigm of comprehensiveness in the curriculum and strategies for nursing education: a cultural-historical approach. Rev. Enferm. Centro O. Min. [Internet]. 2014 [cited 2020 Nov 08]; 4(1):961-70. Available from: http://www.seer.ufsj.edu.br/index.php/recom/article/view/426/571

17. Presidência da República (Brasil). Lei no 9.394, de 20 de dezembro de 1996. Estabelece as diretrizes e bases da educação nacional. [Internet]. Diário Oficial da União. 20 de Dezembro de 1996. [cited 2020 Nov 08]. Available from: http://www.planalto.gov.br/ccivil_03/leis/19394.htm

18. Minayo, MCS (org). O desafio do conhecimento: pesquisa qualitativa em saúde. 14ạ ed. São Paulo: Hucitec; 2012.

19. Duarte R. Qualitative research: reflections on field work. Cadernos de Pesquisa. [Internet]. 2002 [cited 2020 Nov 08]; 115:13954. Available from: https://www.scielo.br/pdf/cp/n115/a05n115.pdf.

20. 20.Camargo BV, Justo AM. Iramuteq: um software gratuito para análise de dados textuais. Temas psicol. [Internet]. 2013 [cited 2020 Nov 08]; 21(2):513-8. DOI: http://dx.doi.org/10.9788/TP2013.2-16.

21. Justo AM, Camargo BV. Estudos qualitativos e o uso de softwares para análises lexicais. In: Novikoff C, Mithidieri OB (org). Estudos qualitativos e o uso de softwares para análises lexicais. Duque de Caxias: Unigranrio; 2014.

22. Kami MTM, Larocca LM, Chaves MMN, Lowen IMV, Souza VMP, Goto DYN. Working in the street clinic: use of IRAMUTEQ software on the support of qualitative research. Esc. Anna Nery. [Internet]. 2016 [cited 2020 Nov 08]; 20(3):e20160069. Available from: http://www.scielo.br/scielo.php?script=sci_arttext\&pid=S1414-81452016000300213.

23. Zhou W, He G, Wang H, He Y, Yuan Q, Liu D. Job dissatisfaction and burnout of nurses in Hunan, China: a cross-sectional survey. Nurs Health Sci. [Internet]. 2015 [cited 2019 May 21]; 17(4):444-50. DOI: https://doi.org/10.1111/nhs.12213.

24. Araújo MT, Henriques AVB, Velloso ISC, Queiroz CF, Santos AMR. Staff dimensioning of a hospital surgical unit. Rev. Gest. Saúde. [Internet]. 2016 [cited 2019 May 21]; 7(2):650-69. Available from: http://periodicos.unb.br/index.php/rgs/article/view/3519/3206.

25. Volpato M.; Cimbalista S. O processo de motivação como incentivo à inovação nas organizações. Rev. FAE. [Internet]. 2017 [citado em 14 Jun 2019]; 5(3):75-86. Available from: https://revistafae.fae.edu/revistafae/article/view/485/380

26. Pereira T, Bezerra MR, Barros M. Interpersonal relations of the nursing team in the work environment. DêCiência em Foco. [Internet]. 2019 [cited 2019 Sep 21]; 3(1):65-81. Available from: http://revistas.uninorteac.com.br/index.php/DeCienciaemFoco0/article/view/271/82

27. Rocha GSA, Andrade SM, Silva DMR, Terra MG, Medeiros SEG, Aquino JM. Feelings of pleasure of nurses working in primary care. Rev. bras. enferm. [Internet]. 2019 [cited 2019 Sep 21]; 72(4):1036-43. DOI: http://dx.doi.org/10.1590/0034-7167-20180518.

28. Forte ECN, Pires DEP, Martins MMFPS, Padilha MICS, Schneider DG, Trindade LL. Work process: a basis for understanding nursing errors. Rev. Esc. Enferm. USP. [Internet]. 2019 [cited 2019 Sep 21]; 53:e03489. DOI: http://dx.doi.org/10.1590/s1980$220 \times 2018001803489$.

29. Gonçalves FGA. Impacts of neoliberalism on hospital nursing work. Texto \& contexto enferm. [Internet] 2015 [cited 2019 Sep 21]; 24(3):646-53. DOI: http://dx.doi.org/10.1590/0104-07072015000420014.

30. Druck G. The outsourcing in public health: various forms of precarization of labor. Trab. educ. saúde. [Internet] 2016 [cited 2019 Sep 21]; 14(1):15-43. DOI: http://dx.doi.org/10.1590/1981-7746-sol00023.

31. Fernandes HN, Thofehrn MB, Porto AR, Amestoy SC, Jacondino MB, Soares MR. Interpersonal relationships in work if multiprofissional team of Family health unit. J. res: fundam. care online. [Internet] 2015 [cited 2019 Sep 22]; 7(1):1915-26. DOI: https://doi.org/10.9789/2175-5361.2015.v7i1.1915-1926.

32. Peruzzo HE, Silva ES, Batista VC, Haddad MCFL, Peres AM, Marcon SS. Organizational climate and teamwork at the Family Health Strategy. Rev. bras. enferm. [Internet] 2019 [cited 2019 Sep 21]; 72(3):721-7. DOI: http://dx.doi.org/10.1590/0034-71672017-0770.

33. Dejours, C. A loucura do trabalho: estudo de psicopatologia do trabalho. 5a.ed. São Paulo: Cortez; 1998.

34. Santos SVM, Robazzi MLCC, Dalri RCMB, Bardaquim VA, Xavier JJS, Silva LA, et al. B Wellbeing in the working environment in Brazilian nursing schools. Revista Ciencia Y Cuidado. [Internet] 2019 [cited 2019 Sep 21]; 16(2):8-20. DOI:

https://doi.org/10.22463/17949831.1611. 